\section{Disección coronaria espontánea: comunicación de 2 casos tratados quirúrgicamente}

\author{
CECILIA MUÑOZ C., JAVIERA PERELLI S. a, SEBASTIÁN ROBERT B. ${ }^{a}$, \\ DANTE LINDEFJELD, RICARDO ZALAQUETT S.
}

\section{Spontaneous coronary artery dissection. Report of two cases}

\begin{abstract}
Spontaneous coronary artery dissection is a rare condition that usually causes a coronary syndrome, but may also cause sudden death. It is more common in women and is associated with factors such as the peripartum period and oral contraceptive use. We report two cases. A 45-year-old woman with hepatitis $C$, presenting in the emergency room with angina. An intravascular ultrasound showed a dissecting hematoma involving the left, anterior descending and circumflex coronary arteries. She was initially managed with nitroglycerin, anticoagulation and anti-platelet drugs but due to persistence of symptoms, she required surgical revascularization. A 32-year-old woman presenting in the emergency room with angina. A coronary angiogram revealed a dissection of the anterior descending coronary artery. Eight days later an intravenous ultrasound showed a retrograde progression of the dissection and she was subjected to a surgical revascularization.

(Rev Med Chile 2016; 144: 257-261)

Key words: Acute coronary syndrome; Coronary artery bypass grafting (CABG); Percutaneous coronary intervention; Spontaneous coronary artery dissection.
\end{abstract}

División de Enfermedades Cardiovasculares. Facultad de Medicina. Pontificia Universidad Católica de Chile.

anterno de Medicina, Facultad de Medicina. Pontificia Universidad Católica de Chile.

Santiago-Chile.

Recibido el 10 de junio de 2015, aceptado el 25 de noviembre de 2015.

Correspondencia a: Dra. Cecilia Muñoz C. División de Enfermedades Cardiovasculares, Pontificia Universidad Católica de Chile. Marcoleta 367, piso 8. Teléfono: 56223543231 mcmunoz@med.puc.cl
L a disección coronaria espontánea o primaria (DCE) es una causa infrecuente de síndrome coronario agudo (SCA), siendo la causa iatrogénica por instrumentación la más frecuente. En 1931, Pretty, en la autopsia de una mujer de 42 años, reportó como causa de muerte un taponamiento cardiaco por rotura de un aneurisma disecante de la coronaria derecha ${ }^{1}$. Casi 50 años más tarde se realiza el primer diagnóstico angiográfico de disección coronaria. Desde entonces se han publicado mayoritariamente reportes de $\operatorname{casos}^{2}$.

Son dos los mecanismos propuestos para su desarrollo: rotura en la íntima con disección y hemorragia en la capa media y formación de un falso lumen, o hemorragia en la capa media por lesión de vasa vasorum que desencadenaría la disección de la pared ${ }^{3}$.
El origen de esta enfermedad es multifactorial. Entre estos factores se encuentran ser mujer, embarazo o puerperio precoz, uso de anticonceptivos orales, ejercicio físico extremo, trauma torácico, enfermedades inflamatorias sistémicas o del colágeno, espasmo coronario, uso de drogas ilícitas, displasia fibromuscular y origen idiopático ${ }^{3-4}$. La enfermedad aterosclerótica, con rotura de la placa, también puede provocar una DCE; sin embargo, estas son lesiones bien localizadas, debido a atrofia y cicatrización de la media existente que impide la propagación de la disección.

Su presentación clínica es variable, dependiendo de la extensión y severidad de la disección, apareciendo desde un síndrome coronario agudo hasta muerte súbita ${ }^{6}$.

El diagnóstico se hace mediante una corona- 
riografía. Otros métodos, como el ultrasonido intravascular (IVUS) o la tomografía de coherencia óptica (OCT), son útiles para diferenciar la disminución del lumen por un hematoma intramural o una placa aterosclerótica ${ }^{3}$.

Las alternativas de tratamiento incluyen el manejo médico conservador y la revascularización miocárdica percutánea o quirúrgica. Sin embargo, el tratamiento invasivo precoz tiene mejores resultados ${ }^{2}$.

Reportamos dos casos de síndrome coronario agudo por disección coronaria espontánea revascularizados quirúrgicamente.

\section{Caso clínico 1}

Mujer, 45 años, con antecedente de daño hepático crónico por virus hepatitis $\mathrm{C}$ (Child A) y cesárea 3 meses antes. Presentó angina de reposo que cedió espontáneamente. Consultó en servicio de urgencia al tercer día por dolor de mayor intensidad. Al ingreso presentaba signos clínicos de bajo gasto y el electrocardiograma (ECG) mostraba elevación del segmento ST. Hemodinamia de urgencia mostraba aquinesia antero-apical con disminución leve de la función ventricular izquierda, tronco de la coronaria izquierda (TRCI) adelgazado, sin estenosis significativa, similar a una DCE. La sonografía intracoronaria demostró un hematoma disecante que comprometía el TRCI, la descendente anterior, circunfleja y la coronaria derecha desde el tercio medio. La disminución del lumen llegaba hasta 90\% en la descendente posterior. Por extensión de la enfermedad se decidió manejo médico con nitroglicerina, anticoagulación con heparina no fraccionada (HNF) y antiagregación plaquetaria. Se mantuvo monitorizada en unidad coronaria. El ecocardiograma posterior informó disfunción sistólica leve del ventrículo izquierdo (FE 46\%), sin insuficiencias valvulares. Evolucionó con angina de reposo, pero sin compromiso hemodinámico ni cambios electrocardiográficos. Durante la hospitalización se pesquisó pancitopenia (hematocrito $30 \%$, recuento de glóbulos blancos 1.530 y plaquetas 62.600). Cinco días después se repitió la coronariografía que no mostró cambios, pero ante persistencia de angina de reposo se decidió revascularización miocárdica quirúrgica (Figura 1). En la cirugía se encontraron signos claros de disección coronaria en los tres vasos principales con lechos distales normales. Bajo circulación extracorpórea, con canulación aórtica y venosa estándar, hipotermia moderada y cardioplegia cristaloide, se revascularizan los tres territorios con injertos venosos, sin incidentes. En

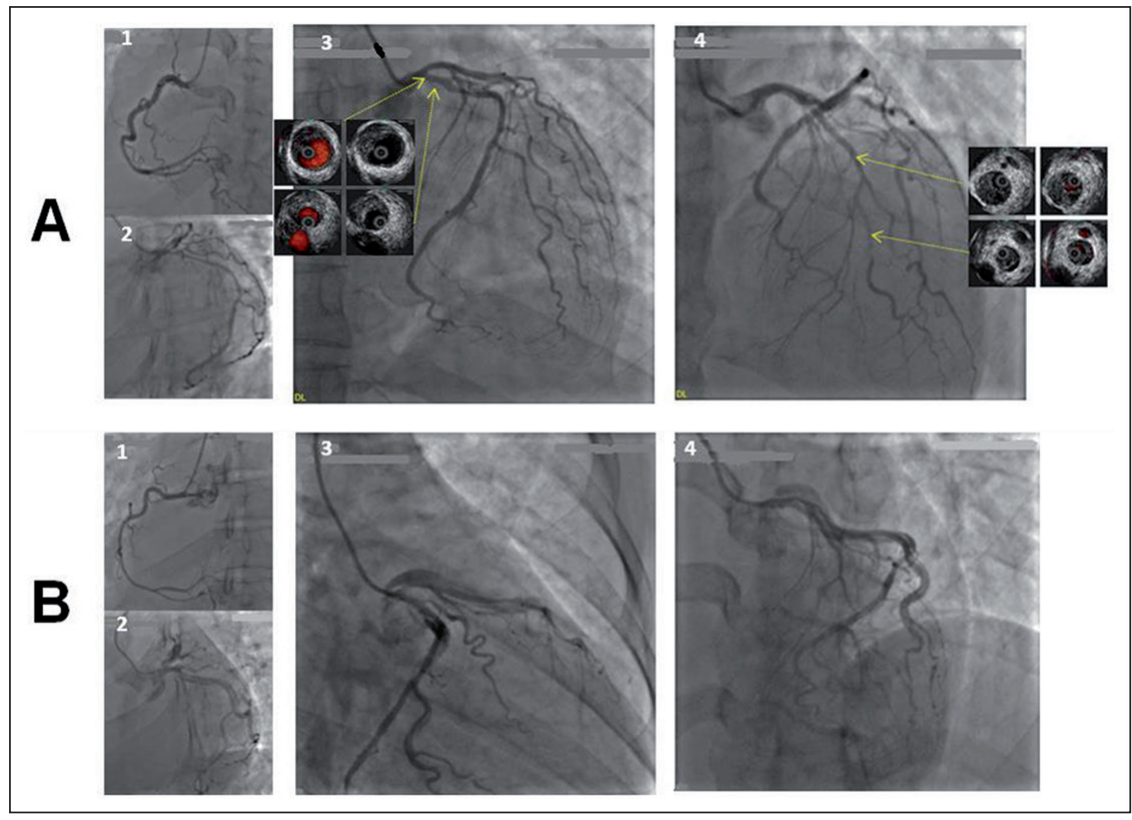

Figura 1. A) Coronariografía inicial: 1. Coronaria derecha con adelgazamiento desde segmento medio que alcanza $90 \%$ a nivel de la descendente posterior. 2. Coronaria izquierda proximal con adelgazamiento difuso. 3. IVUS a nivel TRCI muestra hematoma disecante que compromete descendente anterior y circunfleja. 4. Descendente anterior adelgazada desde su tercio medio y en IVUS hematoma disecante que compromete difusamente la coronaria; B) Coronariografía $5^{\circ}$ día de evolución: 1 y 2 sin cambios. 3 y 4 progresión de las lesiones con mayor compromiso del TRCI y de la descendente anterior por la disección. 
el postoperatorio inmediato presentó hemorragia que requirió re-exploración, reparando satisfactoriamente una filtración de una anastomosis proximal. No presentó otras complicaciones y fue dada de alta en buenas condiciones. En controles no ha referido angina.

\section{Caso clínico 2}

Mujer de 32 años, nuligesta, previamente sana que despertó en la madrugada por angina de reposo, por lo que acudió a servicio de urgencia primario. El primer ECG no mostraba alteraciones, pero una hora más tarde apareció supradesnivel del segmento ST en pared anterior. Se derivó al hospital local, donde se estudió con coronariografía de urgencia que demostró una disección de la arteria descendente anterior a nivel del tercio medio. El ventrículo izquierdo presentaba leve hipocinesia anterior y ápex con función sistólica global conservada. Se decidió manejo médico y anticoagulación con HNF y clopidogrel. No repitió episodios de angina pero la coronariografía de control al $8^{\circ}$ día con IVUS demostró progresión retrógrada de la disección hasta el TRCI, por lo que se decidió tratamiento quirúrgico (Figura 2). En la intervención se observaron signos claros de disección de la arteria descendente anterior hasta su tercio medio. Ante eventual reparación espontánea de la disección, como se describe en la literatura ${ }^{5}$, se decidió revascularización con injertos venosos por la probabilidad de atrofia de conductos arteriales. Utilizando la misma técnica quirúrgica anterior, se revascularizan la descendente anterior y una rama de la circunfleja. No presentó complicaciones del procedimiento y fue dada de alta al $7^{\circ}$ día. En control ambulatorio no ha referido nuevos episodios de angina. Dos meses más tarde, un AngioTAC coronario demostró que los injertos venosos están permeables, la disección estaba cicatrizada y el lumen arterial recuperado.

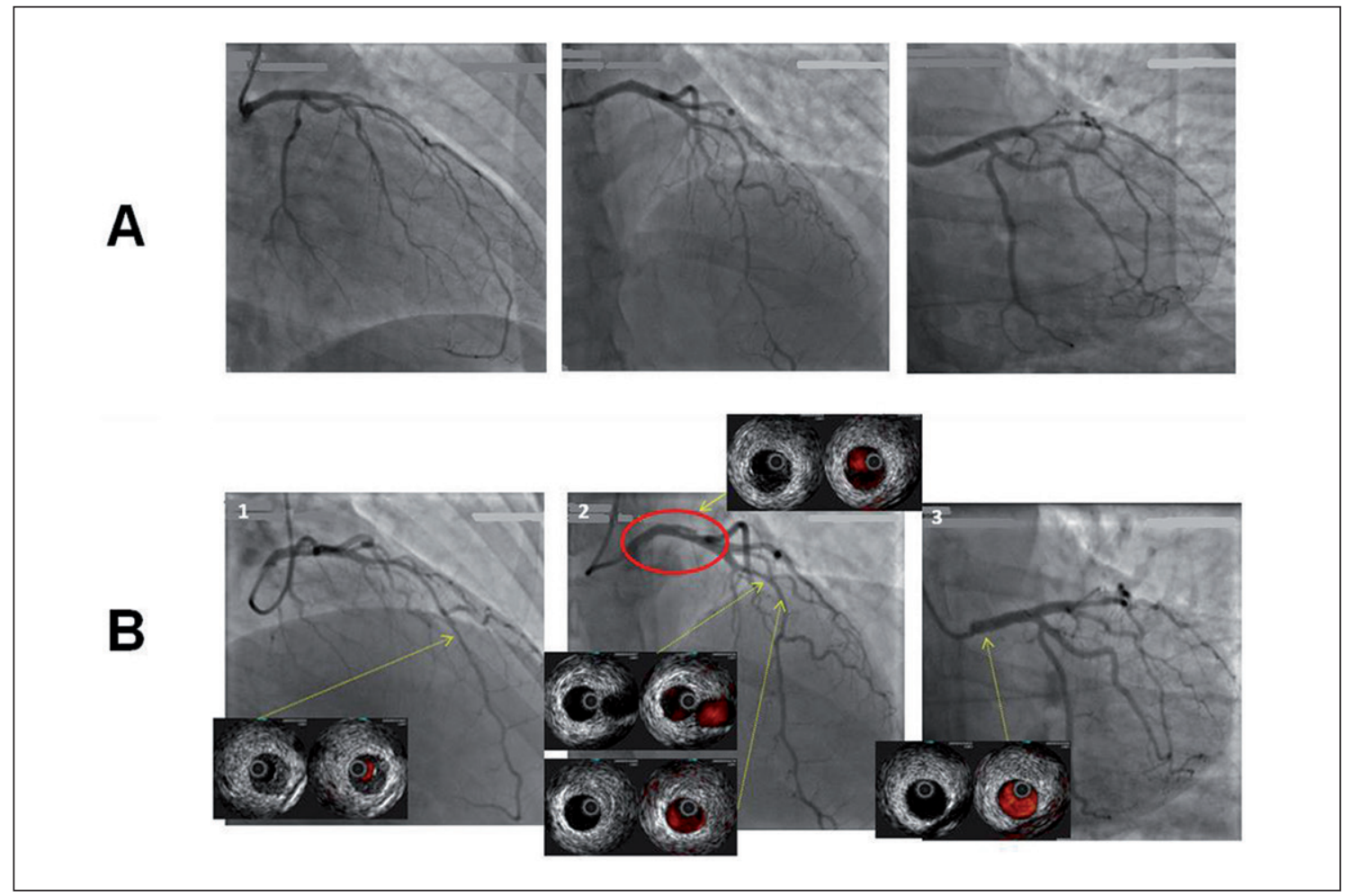

Figura 2. A) Coronariografía inicial: Descendente anterior con adelgazamiento difuso sugerente de hematoma disecante; B) Coronariografía $8^{\circ}$ día evolución: 1. Descendente anterior a nivel tercio medio IVUS con imagen de hematoma disecante. 2. TRCI medio-distal con IVUS que muestra flujo a través de falso lumen y "flap" de disección. Más abajo a nivel de la descendente anterior IVUS muestra consolidación parcial del hematoma. 3. TRCI proximal sin compromiso. 


\section{Discusión}

La DCE se presenta como isquemia miocárdica aguda e incluso como muerte súbita. Su incidencia es baja, aunque su diagnóstico ha aumentado por la mayor frecuencia de coronariografías en síndromes coronarios agudos. Las alternativas de tratamiento van desde el tratamiento conservador hasta la revascularización quirúrgica. No hay acuerdo aún de cuál es el más apropiado y en qué momento realizarlo por el número insuficiente de casos registrados, que impide conocer los resultados de uno u otro. Por lo anterior, la decisión final se hace caso a caso, según la disponibilidad de recursos y la experiencia de los tratantes.

El tratamiento quirúrgico permite la visualización de los lechos libres de enfermedad para realizar la revascularización adecuadamente. La cirugía se ha utilizado en casos de compromiso arterial extenso con isquemia severa o imposibilidad de realizar angioplastía por condición hemodinámica inestable, a pesar de apoyo con balón de contrapulsación intra-aórtico ${ }^{6,7}$. Si no es posible la revascularización, Ferrari ${ }^{8}$ propone incluso apoyo de asistencia ventricular izquierda en espera de recuperación o trasplante cardiaco.

La mejor estrategia de tratamiento es aún incierta. En pacientes con DCE, las paredes de las coronarias serían más frágiles, por lo que lo menos invasivo parece ser mejor, más aún, si son lesiones únicas o distales localizadas con flujo coronario conservado y estabilidad hemodinámica. Una angioplastía podría ser realizada en el mismo momento del diagnóstico y restaurar el flujo coronario. Sin embargo, el sólo intento de realizarla tiene el riesgo de aumentar la extensión de la disección o provocar la oclusión del vaso por el hematoma intramural ${ }^{9,10}$.

La trombolisis en un infarto por disección coronaria espontánea puede aumentar la hemorragia subintimal, incrementando el hematoma disecante y paradojalmente comprometer más la hemodinamia al comprimir el lumen verdadero. La evaluación de los antecedentes del paciente puede llevar a la sospecha clínica de una DCE y evitar una evolución desfavorable ${ }^{11}$.

Los tratamientos no son excluyentes y en algunos casos se asocian todos. En los dos casos que operamos, la presentación clínica característica del SCA permitió el diagnóstico precoz de esta enfermedad con coronariografía. Se intentó inicialmen- te sólo manejo médico, pero por persistencia de los síntomas, en el primer caso, o progresión de la enfermedad, en el segundo, se optó por la cirugía. En ambos casos la revascularización fue completa, sin mortalidad asociada y con buenos resultados a mediano plazo, sin recurrencia de angina a los 2 años de intervenidas. Incluso evidenciándose cicatrización de la disección dos meses después del evento en el caso 2.

En el seguimiento de los pacientes se ha observado cicatrización espontánea hasta en $50 \%$ de los casos, en otros, persistencia de la disección con o sin isquemia residual.

Mucho menos frecuente es el desarrollo de aneurismas o pseudoaneurismas, cuyo tratamiento y pronóstico son muy diferentes. En esos casos, en el diagnóstico más preciso ayuda la sonografía intracoronaria ${ }^{12}$. Para el diagnóstico de estas complicaciones se recomienda realizar un control imagenológico 3 a 6 meses después del evento ${ }^{6}$.

Los reportes de seguimiento a largo plazo de la Clínica Mayo en DCE demuestran recurrencia del síndrome coronario agudo sólo en mujeres ${ }^{9}$. Un primer episodio hasta en $17 \%$ de los casos, un segundo en $29 \%$ y menos frecuente fue un tercer episodio. Las arterias afectadas en las recurrencias, en la mayoría de los casos, no eran las primariamente comprometidas y por el bajo número de pacientes no pudieron hacer una asociación de la recurrencia con los factores de riesgo de DCE o con el tratamiento médico utilizado como prevención secundaria.

\section{Conclusión}

La restauración precoz del flujo coronario en los casos de DCE es el único medio de evitar las peligrosas consecuencias de esta enfermedad. El tratamiento médico y la revascularización percutánea logran en muchos casos este objetivo, sin embargo, la revascularización miocárdica quirúrgica es una alternativa que debe ser considerada y estar disponible ante el fracaso de las medidas anteriores. En algunos casos, puede ser la primera elección de tratamiento ante lesiones extensas y presencia de shock cardiogénico. En estos casos, además de restablecer el flujo coronario, la asistencia circulatoria es otra opción para estabilizar al paciente. 


\section{Referencias}

1. Pretty HC. Dissecting aneurysm of coronary artery in a woman aged 42: rupture. Br Med J1931; 1: 667.

2. Shamloo B, Chintala R, Nasur A, Ghazvini M, Shariat $\mathrm{P}$, Diggs J, et al. Spontaneous coronary artery dissection: aggressive vs conservative therapy. J Invasive Cardiol 2010; 22 (5): 222-8.

3. Saw J. Review Spontaneous Coronary Artery Dissection Canadian Journal of Cardiology 2013; 29: 1027-33.

4. Saw J, Ricci D, Starovoytov A, Fox R, Buller C. Spontaneous Coronary Artery Dissection. Prevalence of Predisposing Conditions Including Fibromuscular Dysplasia in a Tertiary Center Cohort. J Am Coll Cardiol Intv 2013; 6: 44-52.

5. Alfonso F, Paulo M, Lennie V, Dutary J, Bernardo E, Jiménez-Quevedo P, et al. Spontaneous Coronary Artery Dissection. Long-Term Follow-Up of a Large Series of Patients Prospectively Managed With a "Conservative" Therapeutic Strategy. J Am Coll Cardiol Intv 2012; 5: 1062-70.

6. Motreff P, Souteyrand G, Dauphin C, Eschalier R, Cassagnes J, Lusson JR. Management of Spontaneous Coronary Artery Dissection: Review of the Literature and Discussion Based on a Series of 12 Young Women with Acute Coronary Syndrome. Cardiology 2010; 115:
$10-8$.

7. Xin-He Y, Cheng-Jian Y, Yan J, Xin X, Jia-Ning C, Zhen-Jie $Y$, et al. Case Report: A successful emergency management of spontaneous coronary artery dissection and review of the literature. Am J Emerg Med 2013; 31 (7): 1156.e1-1156.e3.

8. Ferrari E, Tozzi P, von Segesser L. Case report: Spontaneous coronary artery dissection in a young woman: from emergency coronary artery bypass grafting to heart transplantation. Eur J Cardiothorac Surg 2005; 28 (2): 349-51.

9. Tweet M, Hayes S, Pitta S, Simari R, Lerman A, Lennon $\mathrm{R}$, et al. Clinical Features, Management, and Prognosis ofSpontaneous Coronary Artery Dissection. Circulation 2012; 126: 579-88.

10. Tokura M, Taguchi I, Kageyama M, Nasuno T, Nishiyama Y, Koshiji N, et al. Clinical features of spontaneous coronary artery dissection. Journal of Cardiology 2014; 63: 119-22.

11. Buys EM, Suttorp MJ, Morshuis WJ, Plokker HW. Extension of a spontaneous coronary artery dissection due to thrombolytic therapy. Cathet Cardiovasc Diagn 1994; 33 (2): 157-60.

12. Agel R, Zoghbi G, Iskandrian A. Spontaneous Coronary Artery Dissection, Aneurysms, and Pseudoaneurysms: A Review. Echocardiography 2004: 21 (2): 175-82. 